\title{
Calcul des réseaux collectifs d'irrigation avec limitation de la demande
}

\author{
J. M. Revuelta Prieto
}

Dr. Ingeniero Agronomo, Pamplona

Nous rappellerons brièvement la méthode de CLEMENT pour le calcul des débits d'un réseau d'irrigation avec distribution à la demande.

Soit $N$ le nombre de prises d'une zone, chacune d'elles peut être ouverte avec une probabilité $p$. Soit donc $q=1-p$ la probabilité qu'une prise quelconque soit fermée.

Dans ce cas le développement du binôme :

$$
(p+q)^{n}=\sum_{i=0}^{i=n}\left(\begin{array}{c}
n \\
i
\end{array}\right) p^{i} q^{n-i}=1
$$

nous donne, dans chacun de ses termes, la probabilité $p$ du fonctionnement de $i$ prises d'un branchement, ou groupement de branchements, qui contient seulement $n$ prises sur le total des $N$ prises du réseau.

La probabilité du fonctionnement de $k$ prises ou moins, sera alors :

$$
P_{k}=\sum_{i=0}^{i=k}\left(\begin{array}{c}
n \\
i
\end{array}\right) p^{i} q^{n-i} .
$$

Quand nous ne cherchons pas la certitude absolue, mais plutôt une probabilité de fonctionnement raisonnable, nous obtenons de très bons résultats, en dimensionnant le branchement, ou le groupement de branchements, pour un fonctionnement simultané de $k$ prises, avec une économie considérable par rapport aux $n$ prises qui garantissent la sécurité totale.
Le calcul de chacun des termes de (2), et leur addition, jusqu'à égaler ou dépasser la probabilité exigée, est assez laborieux. Mais, comme la théorie statistique nous l'indique, la distribution de probabilité binomiale $(p+q)^{n}=1$, dont le terme général est $\left(\begin{array}{c}n \\ i\end{array}\right)=p^{i} q^{n-i}$ peut être approchée très correctement par la distribution normale centrée réduite

$$
f(x)=\frac{1}{\sqrt{2 \pi}} e^{-\frac{x^{2}}{2}} .
$$

Quand $n$ est grand, et $p$ ou $q$ pas trop petits, l'intégrale

$$
F(k)=\frac{1}{\sqrt{2 \pi}} \int_{-\infty}^{k} e^{-\frac{x^{2}}{2}} \mathrm{~d} x
$$

donne une très bonne approximation de l'expression (2).

La fonction (4) est tabulée et elle est d'une grande utilité, pas seulement comme approximation de la distribution binomiale, mais aussi pour la majorité des distributions unimodales.

Nous savons que pour la distribution binomiale

la moyenne $\mu=n p$

la variance $\sigma^{2}=n p q$

et l'écart-type $s=\sqrt{\sigma^{2}}=\sqrt{n p q}$.

\section{Calculating collective irrigation networks with demand limitations}

The Clément method cannot be applied when water demand is limited by the flow rate granted. In this case, binomial distribution of probabilities which justifies the Clément formula, must be replaced by real distribution, in this case, hypergeometric distribution, which is offered as being mathematically adjusted to this situation. 


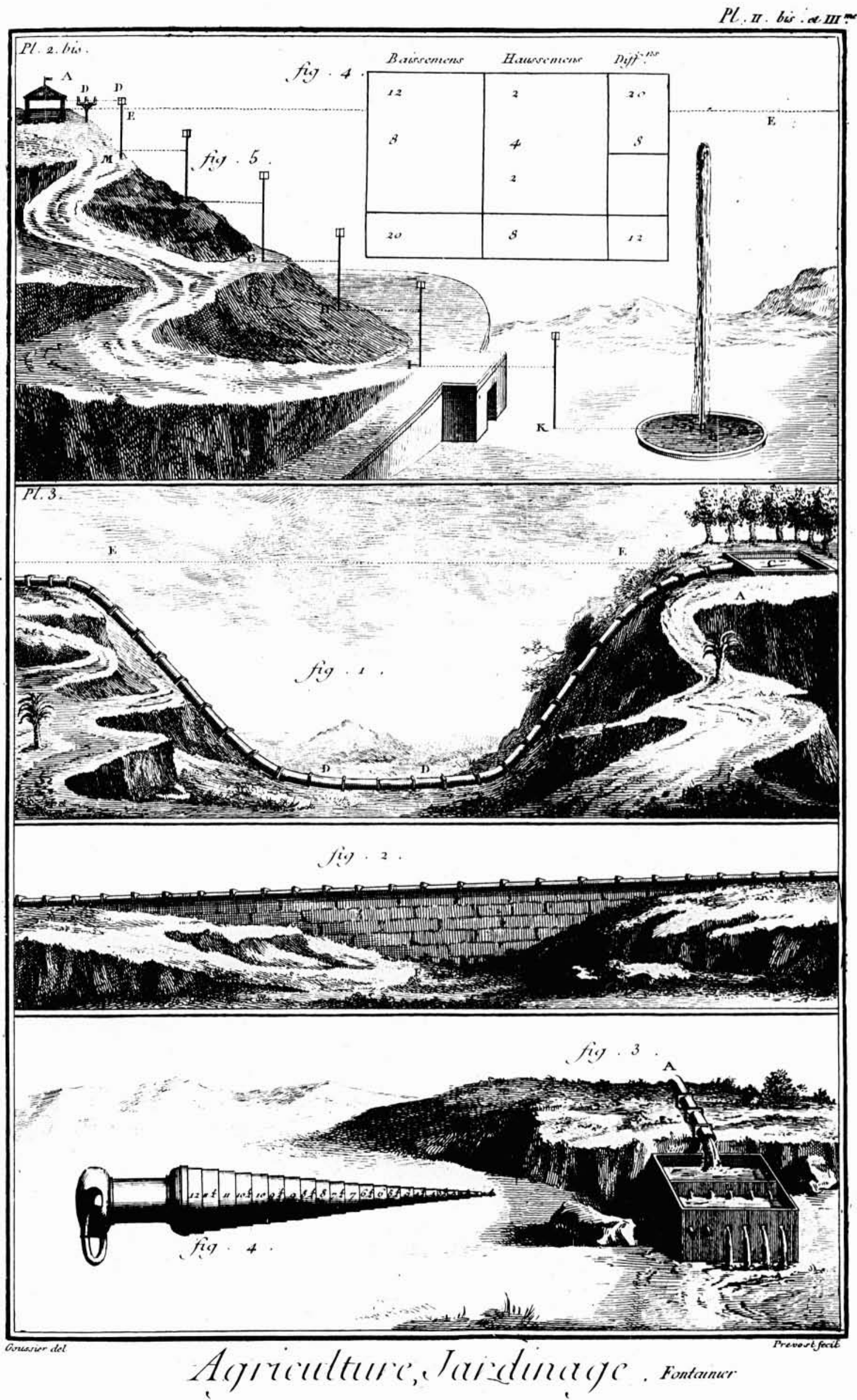


Le nombre $k$ de prises avec lequel on doit calculer un branchement, ou groupe de $n$ prises, de façon à garantir une probabilité de fonctionnement supérieure à $F(x)$, (rappelons que $F(x)$ représente la probabilité cumulée), se calcule en accord avec la théorie statistique, en prenant $x$ de la table de valeurs de l'intégrale $F(x)$ :

$$
k=\mu+x s
$$

ce qui pour la distribution binomiale donnera :

$$
k=n p+x \sqrt{n p q}
$$

qui est la formule de ClémenT.

René CLÉMENT eut l'habileté d'appliquer les résultats obtenus par la théorie statistique au cas pratique du calcul de réseaux collectifs d'irrigation avec distribution de l'eau à la demande.

La correspondance entre $x$ et $F(x)$ est tabulée dans tous les manuels de statistique ; nous donnons ci-dessous un résumé des paires de valeurs les plus usuelles.

\begin{tabular}{|l|l|}
\hline \multicolumn{1}{|c|}{$x$} & $F(x)$ \\
\hline$-\infty$ & 0 \\
0 & 0,500 \\
0,126 & 0,550 \\
0,253 & 0,600 \\
0,384 & 0,650 \\
0,534 & 0,700 \\
0,675 & 0,750 \\
& \\
0,842 & 0,800 \\
1,037 & 0,850 \\
1,282 & 0,900 \\
1,645 & 0,950 \\
1,751 & 0,960 \\
1,881 & 0,970 \\
& \\
2,054 & 0,980 \\
2,327 & 0,990 \\
2,365 & 0,991 \\
2,410 & 0,992 \\
2,455 & 0,993 \\
2,510 & 0,994 \\
& \\
2,575 & 0,995 \\
2,650 & 0,996 \\
2,750 & 0,997 \\
2,880 & 0,998 \\
3,090 & 0,999 \\
& \\
\hline & \\
\hline
\end{tabular}

Mais la distribution binomiale, qui conduit à la formule de CLÉMENT, est uniquement valable quand il n'y a pas limitation dans le nombre $k$ de prises qu'on peut ouvrir simultanément. En Espagne, cette limitation existe, puisque les concessions d'eau pourraient difficilement permettre les débits de pointe requis par l'irrigation à la demande. Pour cette raison, il est nécessaire de se poser le problème du calcul du réseau, tenant compte du fait que le nombre de prises qui peuvent s'ouvrir simultanément est limité.

Soient $N$ le nombre total de prises du réseau, $n$ le nombre de prises dans le branchement ou groupement de branchements, $i=0,1,2,3 \ldots, a$, le nombre de prises ouvertes dans le branchement ou groupement de branchements, $a$ le nombre maximum de prises qui peuvent s'ouvrir simultanément dans tout le réseau.

La probabilité que dans le groupe de $n$ prises, $i$ prises soient simultanément ouvertes (les $a-i$ prises restantes étant ouvertes dans le groupe complémentaire de $N-n$ prises), on obtiendra en divisant le produit du nombre de façons différentes d'ouvrir $i$ prises choisies entre $n$, par le nombre de façons d'ouvrir $a-i$ prises parmi le reste $N-n$, qui est $\left(\begin{array}{c}n \\ i\end{array}\right)\left(\begin{array}{c}N-n \\ a-i\end{array}\right)$, par le nombre de façons d'ouvrir $a$ prises choisies entre $N$, qui est $\left(\begin{array}{c}N \\ a\end{array}\right)$, c'est-à-dire

$$
p_{i}=\frac{\left(\begin{array}{c}
n \\
i
\end{array}\right)\left(\begin{array}{c}
N-n \\
a-i
\end{array}\right)}{\left(\begin{array}{c}
N \\
a
\end{array}\right)}
$$

La probabilité que dans le groupe de $n$ prises soient ouvertes un nombre $k$ de prises sera :

$$
P_{k}=\sum_{i=0}^{i=K} \frac{\left(\begin{array}{c}
n \\
i
\end{array}\right)\left(\begin{array}{c}
N-n \\
a-i
\end{array}\right)}{\left(\begin{array}{c}
N \\
a
\end{array}\right)}
$$

Ce problème, posé et résolu depuis longtemps, quoique exprimé à la manière classique, avec des boules blanches et noires, correspond à celle qu'on appelle distribution hypergéométrique, dont la moyenne, la variance, et l'écart-type sont respectivement

$$
\begin{aligned}
\mu & =n p \\
\sigma^{2} & =n p q \frac{N-n}{N-1} \\
s & =\sqrt{\sigma^{2}}=\sqrt{n p q \frac{N-n}{N-1}}
\end{aligned}
$$

avec $p=a / N$.

L'approximation de l'expression (7) par la distribution normale est excellente, et donne

$$
k=n p+x \sqrt{n p q \frac{N-n}{N-1}}
$$

ce qui est la formule cherchée pour le calcul de divers branchements du réseau. L'expression (8) est d'une précision telle, qu'on doit utiliser la valeur de $k$ arrondie, par défaut ou par excès, selon la règle ordinaire.

La formule de BoIssEzon HaIT, par analogie, se transforme ainsi

$$
k=\sum_{i=1}^{i=n} P_{i} Q_{i}+x \sqrt{\frac{N-n}{N-1} \sum_{i=1}^{i=n} P_{i} q_{i} Q_{i}} .
$$




\begin{tabular}{|c|c|c|c|c|c|c|c|c|c|c|c|}
\hline \multicolumn{4}{|c|}{$\begin{array}{c}F(x)=0,990 \\
x=2,327\end{array}$} & \multicolumn{4}{|c|}{$\begin{array}{c}F(x)=0,900 \\
x=1,282\end{array}$} & \multicolumn{4}{|c|}{$\begin{array}{c}F(x)=0,800 \\
x=0,842\end{array}$} \\
\hline$n$ & (7) & (8) & (8)bis & $n$ & (7) & (8) & (8)bis & $n$ & (7) & (8) & (8)bis \\
\hline 2 & 2 & 2,10 & 2 & 2 & 1 & 1,43 & 1 & 2 & 1 & 1,14 & 1 \\
\hline 4 & 3 & 3,30 & 3 & 4 & 2 & 2,36 & 2 & 4 & 2 & 1,96 & 2 \\
\hline 6 & 4 & 4,35 & 4 & 6 & 3 & 3,20 & 3 & 6 & 3 & 2,72 & 3 \\
\hline 8 & 5 & 5,31 & 5 & 8 & 4 & 4,00 & 4 & 8 & 3 & 3,45 & 3 \\
\hline 10 & 6 & 6,22 & 6 & 10 & 5 & 4,77 & 5 & 10 & 4 & 4,16 & 4 \\
\hline 15 & 8 & 8,33 & 8 & 15 & 7 & 6,61 & $7^{\circ}$ & 15 & 6 & 5,88 & 6 \\
\hline 20 & 10 & 10,29 & 10 & 20 & 8 & 8,36 & 8 & 20 & 7 & 7,55 & $8^{*}$ \\
\hline 25 & 12 & 12,14 & 12 & 25 & 10 & 10,06 & 10 & 25 & 9 & 9,18 & 9 \\
\hline 30 & 14 & 13,91 & 14 & 30 & 12 & 11,71 & 12 & 30 & 11 & 10,78 & 11 \\
\hline 40 & 17 & 17,25 & 17 & 40 & 15 & 14,89 & 15 & 40 & 14 & 13,90 & 14 \\
\hline 50 & 20 & 20,36 & 20 & 50 & 18 & 17,95 & 18 & 50 & 17 & 16,94 & 17 \\
\hline 60 & 23 & 23,25 & 23 & 60 & 21 & 20,89 & 21 & 60 & 20 & 19,90 & 20 \\
\hline 70 & 25 & 25,91 & $26^{*}$ & 70 & 24 & 23,71 & 24 & 70 & 23 & 22,78 & 23 \\
\hline 80 & 28 & 28,29 & 28 & 80 & 26 & 26,36 & 26 & 80 & 26 & 25,55 & 26 \\
\hline 90 & 30 & 30,22 & 30 & 90 & 29 & 28,77 & 29 & 90 & 28 & 28,16 & 28 \\
\hline 100 & 30 & 30,00 & 30 & 100 & 30 & 30,00 & 30 & 100 & 30 & 30,00 & 30 \\
\hline
\end{tabular}

\begin{tabular}{|c|c|c|c|c|c|c|c|c|c|c|c|}
\hline \multicolumn{4}{|c|}{$\begin{array}{c}F(x)=0,700 \\
x=0,534\end{array}$} & \multicolumn{4}{|c|}{$\begin{array}{c}F(x)=0,600 \\
x=0,253\end{array}$} & \multicolumn{4}{|c|}{$\begin{array}{l}F(x)=0,500 \\
x=0,000(1)\end{array}$} \\
\hline$n$ & (7) & (8) & (8)bis & $n$ & (7) & (8) & (8)bis & $n$ & (7) & (8) & (8)bis \\
\hline 2 & 1 & 0,94 & 1 & 2 & 1 & 0,76 & 1 & 2 & 1 & 0,60 & 1 \\
\hline 4 & 2 & 1,68 & 2 & 4 & 1 & 1,43 & 1 & 4 & 1 & 1,20 & 1 \\
\hline 6 & 2 & 2,38 & 2 & 6 & 2 & 2,08 & 2 & 6 & 2 & 1,80 & 2 \\
\hline 8 & 3 & 3,07 & 3 & 8 & 3 & 2,72 & 3 & 8 & 2 & 2,40 & 2 \\
\hline 10 & 4 & 3,74 & 4 & 10 & 3 & 3,35 & 3 & 10 & 3 & 3,00 & 3 \\
\hline 15 & 5 & 5,38 & 5 & 15 & 5 & 4,92 & 5 & 15 & 4 & 4,50 & 4 \\
\hline 20 & 7 & 6,98 & 7 & 20 & 6 & 6,47 & 6 & 20 & 6 & 6,00 & 6 \\
\hline 25 & 8 & 8,56 & $9 *$ & 25 & 8 & 8,00 & 8 & 25 & 7 & 7,50 & 7 \\
\hline 30 & 10 & 10,13 & 10 & 30 & 9 & 9,53 & $10^{*}$ & 30 & 9 & 9,00 & 9 \\
\hline 40 & 13 & 13,20 & 13 & 40 & 13 & 12,57 & 13 & 40 & 12 & 12,00 & 12 \\
\hline 50 & 16 & 16,23 & 16 & 50 & 16 & 15,58 & 16 & 50 & 15 & 15,00 & 15 \\
\hline 60 & 19 & 19,20 & 19 & 60 & 19 & 18,57 & 19 & 60 & 18 & 18,00 & 18 \\
\hline 70 & 22 & 22,13 & 22 & 70 & 22 & 21,53 & 22 & 70 & 21 & 21,00 & 21 \\
\hline 80 & 25 & 24,98 & 25 & 80 & 24 & 24,47 & 24 & 80 & 24 & 24,00 & 24 \\
\hline 90 & 28 & 27,74 & 28 & 90 & 27 & 27,35 & 27 & 90 & 27 & 27,00 & 27 \\
\hline 100 & 30 & 30,00 & 30 & 100 & 30 & 30,00 & 30 & 100 & 30 & 30,00 & 30 \\
\hline
\end{tabular}

(1) Observons que $F(x)=0,500$ et $x=0,000$ n'est autre chose que l'irrigation par roulement, où $k=n p$. 
Un exemple éclairera le procédé à suivre pour le calcul : supposons une zone d'irrigation alimentée par un débit de 300 litres/seconde distribué à travers un réseau doté de 100 prises d'eau, chacun d'eux étant réglé pour un module de 10 litres/seconde. Il est clair que la demande est limitée par un maximum de 30 prises d'eau en fonctionnement simultané. Supposons aussi que la probabilité (ou qualité) du fonctionnement avec lequel on désire calculer le réseau est de 0,990 . Alors

$$
\begin{gathered}
N=100 ; a=300 / 10=30 ; p=a / N=0,30 \\
q=1-p=0,70 .
\end{gathered}
$$

Sur la table de la fonction (4), on voit que pour $F(x)=0,990$, la valeur correspondante de $x$ est égale à 2,327 , nous pouvons appliquer la formule (8) pour n'importe quelle valeur de $n$, par exemple pour un branchement avec $n=20$ prises d'eau.

$$
\begin{aligned}
k & =n p+x \sqrt{n p q \frac{N-n}{N-1}} \\
& =20 \times 0,3+2,327 \sqrt{20 \times 0,3 \times 0,7 \frac{100-20}{100-1}} \\
& =10,29 \approx 10 .
\end{aligned}
$$

Le débit à l'entrée du branchement sera le produit du nombre de prises en fonctionnement simultané par le module de 10 litres/seconde, avec lequel on a réglé chacun d'eux, c'est-à-dire $10 \times 10=100$ litres/seconde.

Si nous développions l'expression (7), nous verrions que pour $k=9$ la qualité ou probabilité du fonctionnement n'arriverait pas à 0,990 , mais se tiendrait à 0,969 , alors que pour $k=10$, la qualité du fonctionnement dépasse déjà la condition exigée, atteignant jusqu’à 0,992 .

Nous donnons dans les tableaux de la page précédente un résumé des valeurs de $k$ obtenues avec ce même exemple pour différentes valeurs de $n$ et pour diverses valeurs de la qualité du fonctionnement.
Comme on peut le voir, l'approximation de l'expression (7) par la formule (8) est aussi précise que celle de la formule (2) par la formule (5) de CLÉMENT.

Les valeurs signalées sur un astérisque montrent de petites déviations par rapport aux valeurs exactes indiquées dans la colonne (7). La colonne (8) bis est la valeur adoptée pour $k$ comme valeur arrondie, selon la règle ordinaire, du chiffre obtenu en employant la formule (8). Les différences entre les colonnes (7) et (8) bis, le sont par excès. Elles sont peu nombreuses et correspondent à des différences entre les qualités de fonctionnement pratiquement inappréciables.

Le système de majoration de débits suivant un coefficient qui diminue depuis la queue à la tête du réseau, où l'on prend un coefficient égal à l'unité, a toujours buté par manque de fondement mathématique, bien qu'il ait constitué un premier essai pour «soulager " la rigidité propre à l'irrigation par "roulement " et pour éviter la contradiction consistant à appliquer la formule de CLÉMENT quand la demande est limitée par le débit de tête. Quand on l'applique dans ces conditions, on adopte les valeurs de $k$ correspondant à $k \leqslant a$, et on maintient la valeur de $a$ jusqu'à la tête du réseau une fois qu'on a atteint cette dernière valeur. Ceci entraîne quelques conséquences défavorables :

- On aboutit à un surdimensionnement du réseau, surtout dans les branchements les plus éloignés de la tête.

- On obtient des qualités de fonctionnement si basses, à mesure que l'on approche de la tête du réseau, que, quoique fausses, elles justifieraient par cela même l'invalidation de la formule, ou bien elles laisseraient planer un doute sur les véritables qualités de fonctionnement. - Quelquefois aussi, pour "améliorer " la basse qualité du fonctionnement dans la tête du réseau, on abaisse artificiellement la probabilité du fonctionnement de chaque prise, par une augmentation des heures employées pour chaque irrigation, ce qui éloigne encore plus de la méthode, attribuant la même probabilité de fonctionnement aux heures de nuit qu'aux heures diurnes.

\section{Bibliographie}

J. C. Turner, Matemática moderna aplicada, (traduction en espagnol de Andrés Ortega Klein), Alianza Universidad.

Paul G. HotL, Introducción a la estadistica matemática, (traduction en espagnol de Alcjandro Herranz Juste), Ed. Aricl.

V. K. Zajarov, B. A. Sevastianov y V. P. Christiakov, Teoria de la probabilidad, (traduction en espagnol de A. I. Samojvalov), Editorial MIR.

M. Poiree y Ch. Ollier, El regadio, (traduction en espagnol de Francisco Sierra), Editores Técnicos Asociados, S.A.

C. BONNAL, Manual de riego colectivo por aspersión, (traduction en espagnol de J. A. Ortiz y Fdez. de Urrutia), Organización de cooperación y desarrollo cconómico. 